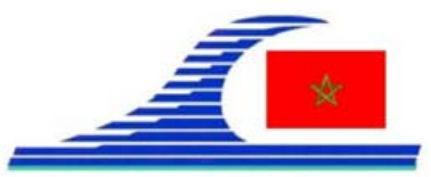

Conférence Méditerranéenne Côtière et Maritime

EDITION 2, TANGER, MAROC (2011)

Coastal and Maritime Mediterranean Conference

Disponible en ligne - http://www.paralia.fr - Available online

\title{
Modélisation hydrodynamique de la nappe de Rhis-Nekor (Maroc) et impact de l'intrusion marine
}

\author{
Nisrine IOUZZI ${ }^{1}$, Abdelkader LARABI ${ }^{1}$, Mohamed FAOUZI ${ }^{2}$ \\ 1. LIMEN, Ecole Mohammadia d’Ingénieurs, BP 765, Agdal, Rabat, Maroc. \\ iouzzi@mtpnet.gov.ma \\ 2. Laboratoire LGRN, Faculté des Sciences Dhar El Mahraz, BP 1796 (Atlas), Fès \\ 30.000, Maroc.
}

\section{Résumé :}

La nappe de Rhis Nekor est considérée comme la plus importante nappe alluviale de la zone méditerranéenne. Néanmoins, elle est confrontée à des pressions naturelles et anthropiques majeures, notons l'aridité de la région, le développement de l'activité agricole, la vulnérabilité à l'intrusion marine ainsi que son faciès chloruré-sodique et/ou potassique à sulfaté-sodique ce qui constitue un enjeu majeur pour ses ressources en eau.

Le recours à la modélisation hydrogéologique a servi d'outil d'aide à la décision. Le modèle issu de cette étude a permis de simuler le comportement hydrodynamique de la nappe durant une période allant de 1972 à 2007. Les premières années de simulation montre une forte dépendance de la nappe aux sollicitations due à la mise en place du barrage Mohammed Ben Abdelkarim El Khattabi. A partir de cette date, les fluctuations piézométriques de la nappe sont fortement dépendantes au régime des précipitations et aux autres sources d'apport. Les scénarios établis ont permis de faire des simulations futures (jusqu'à 2027) traduisant les sollicitations diverses auxquelles la nappe pourrait être confrontées, d’où une éventuelle intrusion marine qui se met en place.

\section{Mots clés :}

Hydrogéologie - Nappe de Rhis Nekor - Intrusion marine - Modélisation - Scénarios

\section{Introduction}

La nappe de Rhis Nekor circule dans un remplissage quaternaire, constitué d'alluvions hétérogènes. De forme triangulaire, elle s'étend sur une superficie d'environ $100 \mathrm{~km}^{2}$ entre la mer Méditerranée au nord, le massif calcaire des Bokoya au nord-ouest, les vulcanites au nord-est, les cônes de déjection à l'est et par les flyschs schisto-gréseux imperméables au sud-est.

Elle est en effet traversée par deux principaux cours d'eau et recèle la plus importante nappe alluviale de la région. De point de vue agricole, la région comprend les meilleures terres cultivables et elle abrite les plus importantes concentrations de populations et les principales agglomérations dont la ville d'El Hoceima. 
La connaissance de la Mer :

un vecteur du développement durable en Méditerranée

L'aménagement hydraulique de cette plaine, dont le barrage Mohamed Ben Abdelkrim El Khattabi constitue la pièce maîtresse de son évolution, ce qui a contribué d'une manière significative à la promotion et au développement socio-économique de cette région qui s'est transformée depuis 1981, date de mise en service de cet important ouvrage.

Le climat de la plaine de Rhis-Nekor est de type semi-aride caractérisé par un été chaud et sec et auquel succède une saison pluvieuse fraiche, et conditionné par deux facteurs : L’influence maritime et l'existence de la chaîne de montagnes du Rif.

La reconnaissance de l'aquifère a été fortement étudiée grâce à la campagne géophysique réalisée en 2003 et qui a couvert toute la plaine (ONEP,2004).

D’après le diagramme de PIPER, le faciès chimique de la nappe de Rhis-Nekor a été élaboré grâce aux résultats de la campagne de prélèvements et d'analyse chimique réalisée par en 2005, il s'avère que ledit faciès est chloruré-sodique et potassique à sulfaté-sodique et lié spécialement aux eaux de la mer, et au lessivage des terrains adjacents (figure 2).

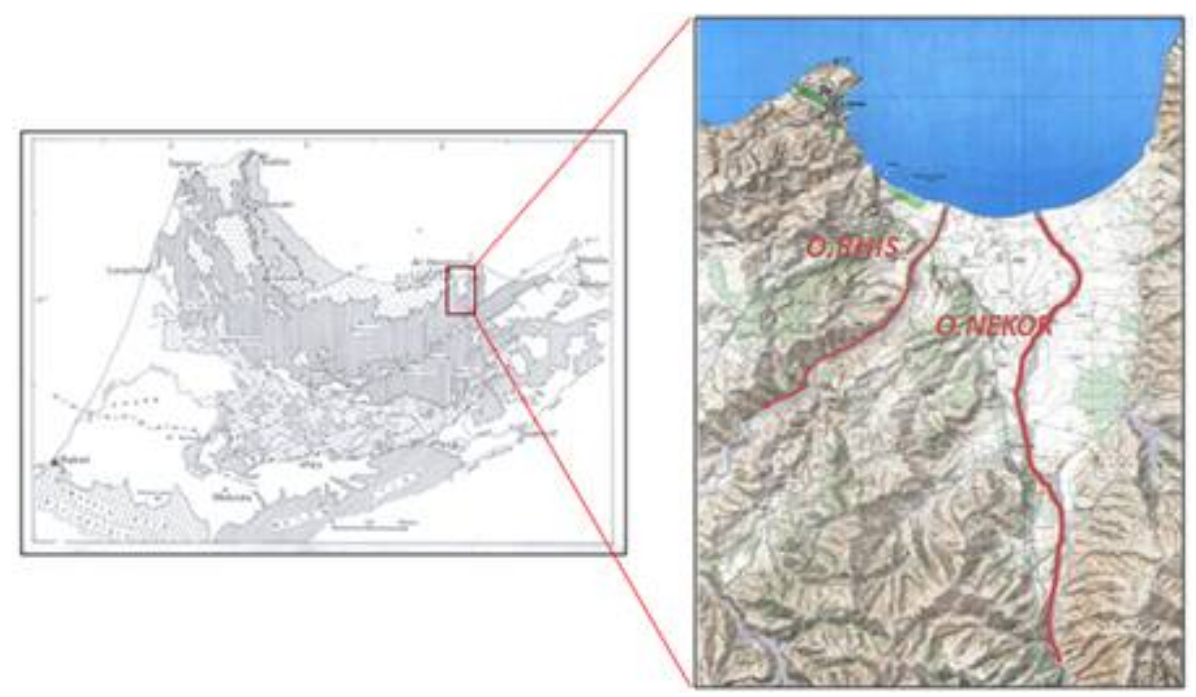

Figure 1. Situation de la zone d'étude.

\section{Méthodologie}

Le système aquifère de Rhis Nekor est discrétisé en mailles régulières de 250 mètres de côté, soit 54 colonnes et 66 lignes. Le nombre total de mailles est de 3564 dont 1285 mailles actives, et 198 mailles frontières.

L'aquifère est constitué par des alluvions d’âge plio-quaternaire, il a été introduit en une seule couche homogène. D'après les coupes effectuées $\mathrm{N}-\mathrm{S}$, l'aquifère présente une variation d'épaisseur remarquable, il devient plus épais vers l'aval. La coupe E-W passant d'Imzouren montre une dépression importante où il atteint le maximum de profondeur allant à $240 \mathrm{~m}$, ajoutons également une dépression relevée dans la corne Ouest de la zone avale JOUNAID (2002). 
A best knowledge of the Sea:

A sustainable development vector in Mediterranean

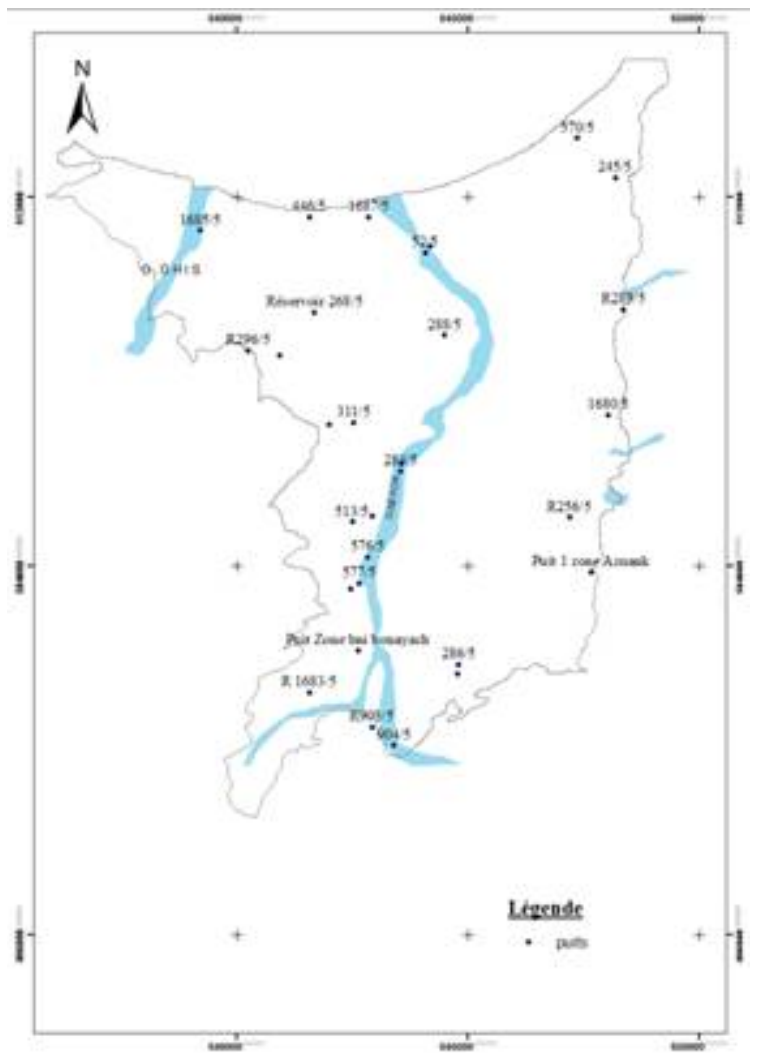

Figure 2.a Situation des points de prélèvement (à droite).

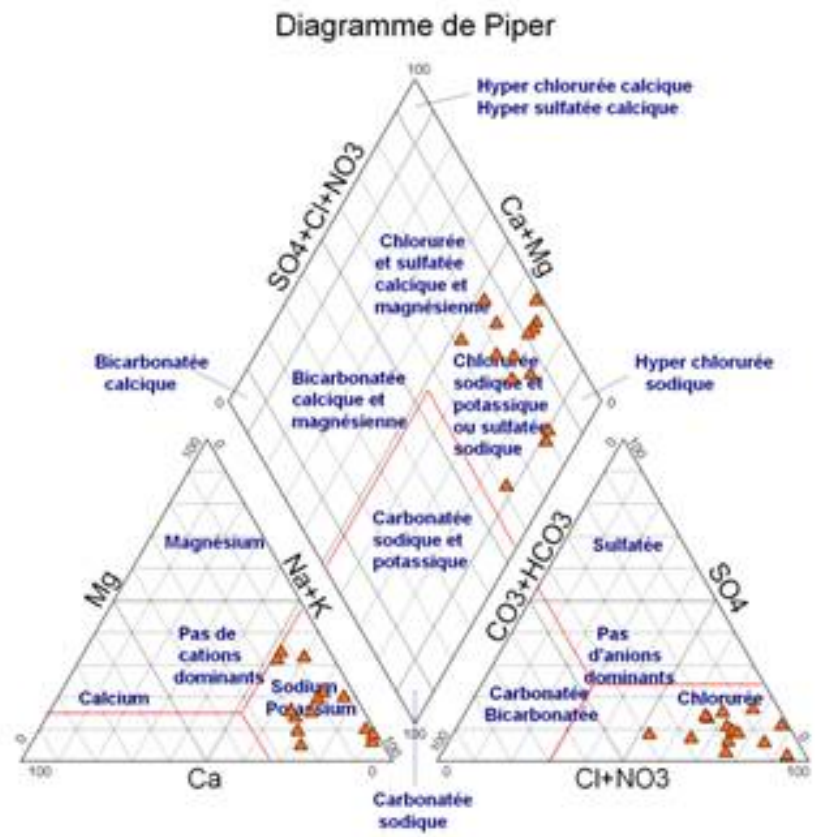

Figure 2.b. Représentation des analyses chimiques de la nappe de Rhis Nekor dans le diagramme de Piper. 
La connaissance de la Mer :

un vecteur du développement durable en Méditerranée

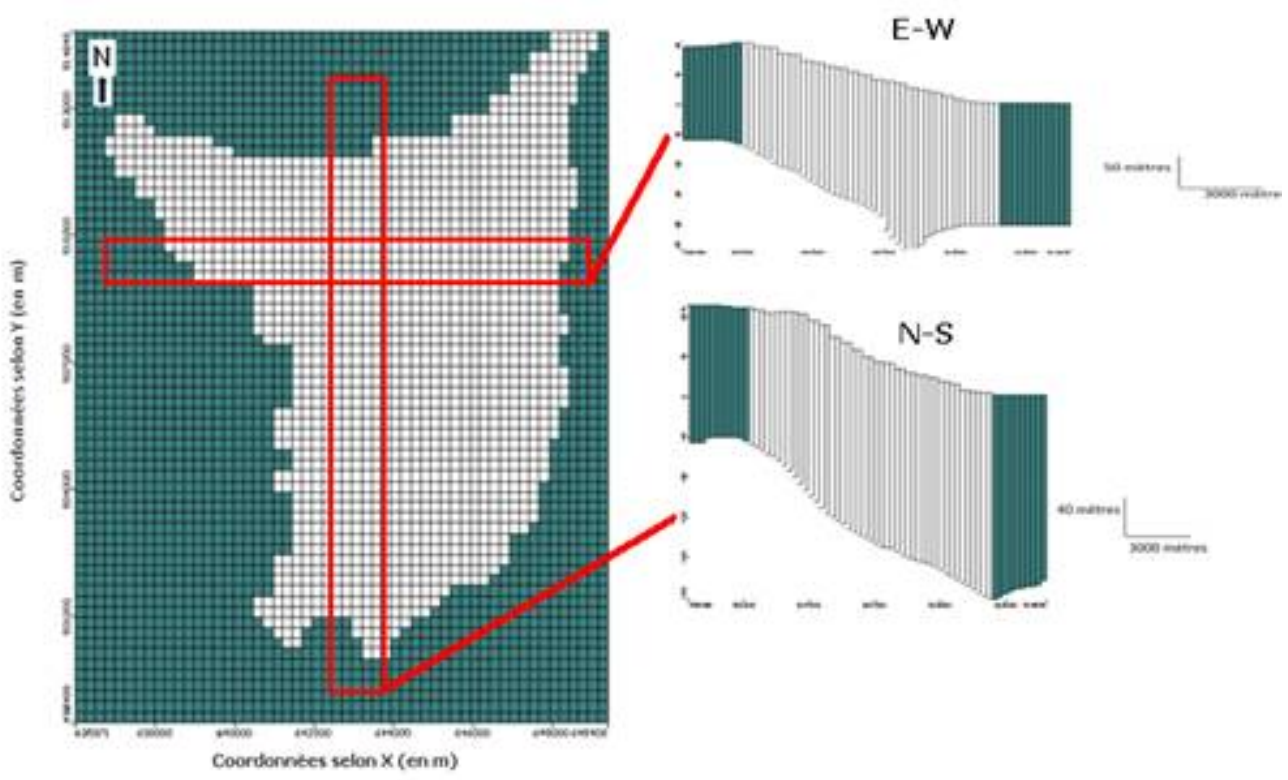

Figure 3. Discrétisation du domaine en différences finies.

\section{Principaux résultats}

Le calage en régime permanent a été réalisé par la calibration des paramètres de perméabilités. L'état de référence pris est celui de l'année 1973 (THAUVIN, 1971). L'examen du graphe de calibration montre une bonne concordance entre la piézométrie calculée et celle mesurée sur le terrain. L'écart entre les deux piézométries ne dépasse pas 1 mètre pour la majorité des points d’observation (Fig. 4).
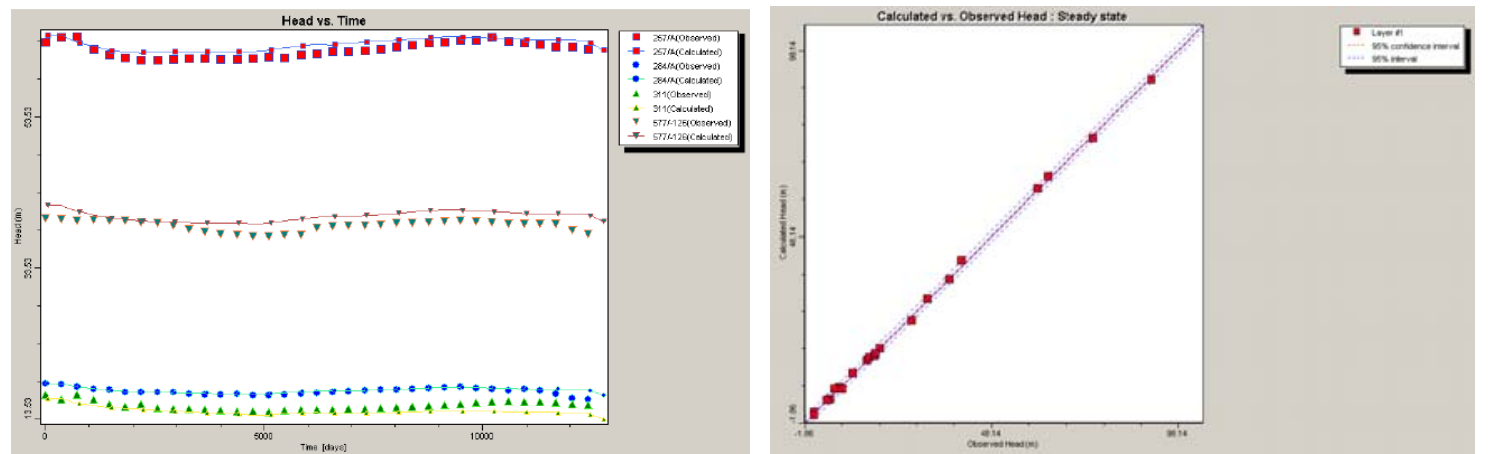

Figure 4. Piézométries calculées et mesurées en Régime Transitoire (à gauche) Corrélation entre piézométries calculées et mesurées en Régime Permanent (à droite). 

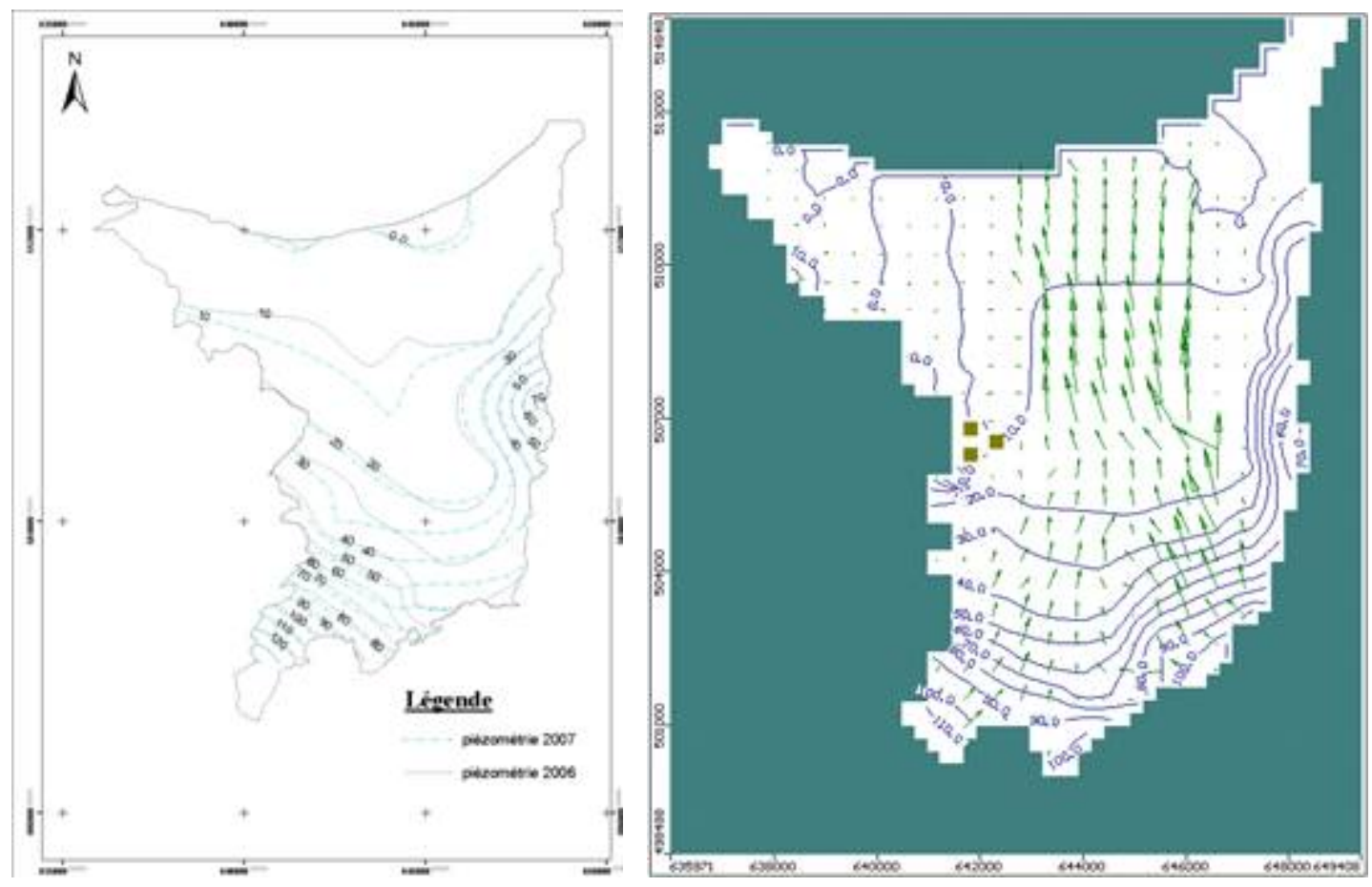

Figure 5. Cartes piézométriques comparatives de la nappe de Rhis Nekor des années 2006 et 2007(à gauche) - Carte piézométrique de l'année 2025 dans le cas de multiplication de pompage par 5 (en mètres) (à droite).

Le calage du modèle en Régime Transitoire (RT) a donné un écart satisfaisant ne dépassant pas 1 mètre dans les piézomètres de contrôle (Fig. 4).

Trois scénarios ont été effectués pour connaître le comportement futur de la nappe suite à des sollicitations futures. Les résultats donnés par le modèle pour les scénarios liés à l'intensification des pompages sont les suivants :

- Pour des pompages multipliés par 2 : rabattement moyen de la nappe de $1 \mathrm{~m}$.

- Pour des pompages multipliés par 3 : rabattement moyen de la nappe de $2 \mathrm{~m}$.

- Pour des pompages multipliés par 5 : rabattement moyen de la nappe de $4 \mathrm{~m}$ avec des zones où on note des mailles sèches, ainsi qu’une éventuelle intrusion marine (Fig. 5).

\section{Références bibliographiques}

JOUNAID H. (2002). Hydrogéologie de la nappe de Rhis Nekor. Thèse de doctorat en sciences appliquées, Ecole Mohammadia d’Ingénieurs.

ONEP (2004). Etude géophysique par la méthode électrique dans la nappe de Rhis Nekor. Géoatlas 2003. 
La connaissance de la Mer :

un vecteur du développement durable en Méditerranée

THAUVIN J.P. (1971). Présentation du domaine rifain. In : Ressources en eau du Maroc : T.1. Domaine du Rif et du Maroc Oriental. Notes et Mémoires du Service Géologique du Maroc, Rabat, n²31, pp 27-67. 